\section{Weather forecasts still tricky}

SIR-The recent paper on 'Fractal characterization of inhomogeneous geophysical measuring networks' by Lovejoy et al. ${ }^{\prime}$ and a discussion in News and Views ${ }^{2}$ address the important problem of detectability of sparse atmospheric phenomena and, therefore, of the very feasibility of useful weather forecasting. Now, whatever the difficulty of its detection might be, a sparse phenomenon will exert an influence on the time course of a multitude of more systematic phenomena occurring over a wide range of space scales. The main reason for this is the fundamental instability of the atmospheric system ${ }^{3}$ : a perturbation arising from an uncontrollable localized event and acting on a larger scale phenomenon pertaining to atmospheric circulation may give rise to an (exponentially) divergent history and to an altogether different regime, or to an aperiodic sequence of regimes perceived by an unaware observer as 'noise'.

Let $\gamma$ be the precision with which the initial state of the system can be determined in phase space. Assuming that the atmosphere behaves as an unstable dynamical system we obtain the following estimate for the error growth after time $t$ :

$$
\gamma(t)=\gamma \mathrm{e}^{t \kappa}
$$

where $K$ is the largest positive Lyapounov exponent (or more generally the Kolmogorov entropy).

Since our variables are distributed in physical space, an inadequate spatial resolution $l$ will clearly induce an error in the estimate of the variables. Conversely, if for some reason their values are subject to uncertainty, it will be difficult to locate with precision the region of space from which these variables had initially emanated. We may therefore substitute, in our crude estimate, the phase space uncertainty $\gamma$ by the precision $l$ with which the initial state of the atmosphere can be determined in the physical space and write, instead of (1)

$$
l(t)=l \mathrm{e}^{\mathrm{t}}
$$

When the size $l(t)$ will reach a value of the order of the dimensions of the entire system, $L$, predictions concerning individual histories will become meaningless and should be replaced by statistical considerations. The time $\tau_{\mathrm{m}}$ for which this will happen is:

$$
\tau_{\mathrm{m}} \simeq \frac{1}{K} \ln \frac{L}{l}
$$

This gives a quantitative measure of the limits of predictability of the system. A significant feature of $\tau_{\mathrm{m}}$ is its logarithmic dependence on the parameter $l$, and thus its relative insensitivity to space resolution. Going from a quasi-uniform to a fractal distribution of $l$ or vice versa is not going to change this picture drastically.

So although detection of sparse phenomena is important in its own right, it is worth stressing that even with a considerable improvement of the world detection network the problem of forecasting of the global state of the atmosphere is likely to remain as acute as ever.

C. Nicolis Institut d'Aéronomie Spatiale de Belgique, 1180 Bruxelles, Belgium

G. NiCOLIS

Faculté des Sciences,

Université Libre de Bruxelles,

1050 Bruxelles, Belgium

1. Lovejoy, S. et al. Nature 319,43-44 (1986).

2. Hollingsworth, S. Nature 319, $11-12(1986)$

3. Lorenz, E.N. Tellus 36A, 98-110 (1984)

4. Schuster, H.G. Deterministic Chaos (Physik, Weinheim, 1984).

\section{Activation of cellular calcium entry}

SIR-A recent News and Views piece by Baker ${ }^{1}$ discusses the mechanisms of intracellular calcium ion $\left(\mathrm{Ca}^{2+}\right)$ release from the endoplasmic reticulum (ER) but refers briefly to cellular calcium entry from the extracellular fluid as being mediated by $\mathrm{Ca}^{2+}$ channels gated either chemically or by voltage. Voltage-activated $\mathrm{Ca}^{2+}$ channels that can be modulated by intracellular messengers have been characterized in many systems ${ }^{2}$, but are unlikely to be present in a number of different epithelial tissues such as exocrine glands and liver simply because they are unable to fire action potentials and many activating hormones or neurotransmitters evoke membrane hyperpolarization rather than depolarization in these cells ${ }^{3}$. Nevertheless there is clear evidence that hormones and neurotransmitters can increase unidirectional $\mathrm{Ca}^{2+}$ flux into, for example, pancreatic acinar cells ${ }^{4}$.

The mechanisms underlying this receptor operated $\mathrm{Ca}^{2+}$ entry are at present obscure although a link with the increased turnover of phospholipids after stimulation has been suspected for more than ten years ${ }^{5}$ with evidence for such a link being particularly clear in relation to $\mathrm{Ca}^{2+}$ entry in blowfly salivary glands ${ }^{6}$. A recently formulated hypothesis ${ }^{7}$ suggests that receptor operated $\mathrm{Ca}^{2+}$ entry is secondary to the emptying of an intracellular $\mathrm{Ca}^{2+}$ pool mediated by the action of inositol trisphosphate $\left(\mathrm{IP}_{3}\right)$ on an electrogenic $\mathrm{Ca}^{2+}$ pathway (channel) in the $\mathrm{ER}^{8.9}$. The well-established biphasic nature of agonist-activated $\mathrm{Ca}^{2+}$ mobilization is, according to the new hypothesis, explained by an initial emptying of the intracellular (ER) $\mathrm{Ca}^{2+}$ pool by $\mathrm{IP}_{3}$ followed by entry of extracellular $\mathrm{Ca}^{2+}$ into the pool and, in the continued presence of $\mathrm{IP}_{3}$, into the cytosol. On removal of the stimulant, $\mathrm{IP}_{3}$ is rapidly broken down closing the $\mathrm{Ca}^{2+}$ channel in the ER and $\mathrm{Ca}^{2+}$ entry from the outside continues only until the content of the ER pool has reached a level that prevents further entry.
In this model ${ }^{7}$ intracellular $\mathrm{Ca}^{2+}$ release and $\mathrm{Ca}^{2+}$ entry are both controlled by the same messenger, $\mathrm{IP}_{3}$. $\mathrm{Ca}^{2+}$ entry is therefore not directly linked to the hormone receptor, but is messenger-mediated. A recent patch-clamp study on pancreatic acinar cells ${ }^{10}$ demonstrating an external $\mathrm{Ca}^{2+}$ requirement for sustained $\mathrm{K}^{+}$ channel $\left(\mathrm{Ca}^{2+}\right.$-activated $)$ opening evoked by a peptide belonging to the cholecystokinin-gastrin group may be relevant in this context since the $\mathrm{Ca}^{2+}$-requirement could be localized at a site separated from the hormone-receptor interaction indicating messenger-mediated rather than receptor-mediated $\mathrm{Ca}^{2+}$ uptake. These recent findings ${ }^{10}$ appear to be compatible with the hypothesis forwarded by Putney ${ }^{7}$.

Ole H. Petersen

MRC Secretory Control Research Group, University of Liverpool, Liverpool L69 $3 B X, U K$

1. Baker, P.F. Nature 320, 395 (1986)

2. Reuter, H. Nature 301, 569-574 (1983)

3. Petersen, O.H. \& Maruyama, Y. Nature 307, 693696 (1984).

4. Kondo, S. \& Schulz, I. Biochim. biophys. Acta 419, 76-92 (1976)

Michell, R.H. Biochim. biophys. Acta 415, 81-147 (1975). 6. Berridge, M.J. \& Fain, J.N. Biochem. J. 178,59-69 (1979). 7. Putney, J.W. Cell Calcium 7, 1-12 (1986).

Streb, H., Irvine, R.F., Berridge, M.J. \& Schulz, I. Nature 306, 67-69 (1983).

Mualem, S., Schoeffield, M., Pandol, S. \& Sachs, G. Proc. natn. Acad. Sci. U.S. A. 82, 4433-4437 (1985).

10. Suzuki, K., Petersen, C.C.H. \& Petersen, O.H. FEBS Lett. 192, 307-312 (1985).

\section{Beam balance test of weak equivalence principle}

SIR-In the recent article by Fischbach et al. ${ }^{1}$, commenting on a News and Views piece $^{2}$, a new hypercharge force is postulated as being responsible for various apparently anomalous effects in physics. Although there may not be unanimity in the interpretation of the Eötvös data in terms of this new force ${ }^{3}$, its existence would have such far reaching consequences in physics that further experiments are clearly required. As suggested by Fischbach et al., further Eötvös-type or galilean experiments come to mind. Here we propose an experiment using a common balance which would allow the force, if it exists, to be measured with good precision. The experiment would be as follows.

The weights of the two test masses made of materials having different values of $(B / \mu)$ (see ref. 1 for notation) are compared using a beam balance at two different sites. The first site is in a tunnel deep inside a mountain where it can be assured that there are some kilometres of solid rock in all directions. Here, in the absence of any net hypercharge force, the test masses are adjusted to have apparently equal masses as measured using the balance. The balance and test masses are then moved out of the tunnel to a second site on the surface of the Earth, preferably 